Ingeniare. Revista chilena de ingeniería, vol. $17 \mathrm{~N}^{\circ} 2,2009$, pp. 213-222

\title{
ANÁLISIS EN EL PLANO R-X PARA LOCALIZAR FALLAS DE ALTA IMPEDANCIA
}

\section{R-X AXIS ANALYSIS TO LOCATE HIGH IMPEDANCE FAULTS}

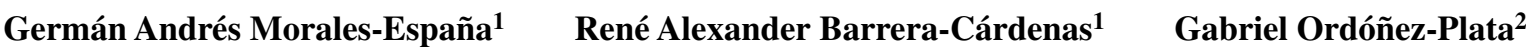 \\ Recibido 8 de septiembre de 2008, aceptado 12 de junio de 2009 \\ Received: September 8, 2008 Accepted: June 12, 2009
}

\begin{abstract}
RESUMEN
Este artículo propone una herramienta de análisis en el plano R-X de un sistema en falla para resolver el problema de localización de fallas en sistemas de potencia. La herramienta permite localizar todo tipo de fallas incluidas las de alta impedancia y fallas en sistemas con sobrecarga. Metodológicamente, se analiza el plano R-X de la impedancia aparente de las fases involucradas en la falla, y utilizando interpolación bidimensional se logra la ubicación de la falla a partir de curvas de distancia previamente obtenidas del sistema mediante simulación. Como resultados se presentan pruebas en un sistema de referencia sometido a los cuatro tipos de falla con diversas resistencias localizadas en diferentes sitios dentro del sistema, resaltándose la obtención de errores inferiores al 3\% para fallas monofásicas y resistencia de falla hasta $1000[\Omega]$.

Palabras clave: Fallas de alta impedancia, impedancia aparente, localización de fallas, plano R-X.
\end{abstract}

\begin{abstract}
This paper proposes an analysis tool using the $R$-X axis of a faulted system to solve the fault location problem in power systems. The proposed approach allows locating all types of faults including high impedance ones and faults on overload systems. Methodologically, the apparent impedance $R$-X axis of faulted phases is analyzed and the fault is located from distance curves, previously obtained from the power system, by using two-dimensional interpolation. As results, tests of a reference system with four types of faults and different fault resistances located on different places on the system are presented. Errors are kept lower than 3\% for single phase faults and fault resistances up to 1000[ 2$]$.
\end{abstract}

Keywords: High impedance faults, apparent impedance, fault location, $R$-X axis.

\section{INTRODUCCIÓN}

Un reto en el ámbito de las protecciones de los sistemas de potencia es la detección y localización de fallas de alta impedancia (HIF: High Impedance Faults) debido a que éstas pueden ser confundidas con cambios en la carga del sistema y tendrá un impacto negativo en la continuidad del servicio. Las causas más comunes de este tipo de fallas en los sistemas de potencia son la generación de arcos eléctricos por desgaste en los aislamientos de la línea y el contacto con objetos cercanos tales como árboles, automóviles, cercas o cualquier superficie cercana al conductor; esto último supone un riesgo potencial para la población ante la posible exposición a electrocución, quemaduras eléctricas e iniciación de incendios por arcos eléctricos o calentamiento de materiales [1-3].
Una falla de alta impedancia se puede confundir con un incremento normal en la carga, dado que la corriente de falla tiene un incremento en la magnitud que algunas veces no es detectado por los dispositivos de sobrecorriente [4]. Se han desarrollado varios estudios enfocados a la detección de este tipo de fallas con el propósito de reducir las condiciones de peligro debidas a la no detección [3, 5-11]. Sin embargo, no solo la detección es importante, sino también su rápida localización.

En el caso de presentarse una falla permanente de alta impedancia, al ser detectada por el sistema de protección, un alimentador puede ser desenergizado y ante tal evento hay dos posibles acciones posteriores:

\footnotetext{
1 Universidad Industrial de Santander (UIS). Grupo de Investigación en Sistemas de Energía Eléctrica (GISEL). E-mail: G.A.MoralesEspana@student.tudelft.nl, german.morales.e@gmail.com; abarrera@ uis.edu.co

2 Universidad Industrial de Santander (UIS). Escuela de Ingeniería Eléctrica, Electrónica y Telecomunicaciones. Grupo de Investigación en Sistemas de Energía Eléctrica (GISEL). E-mail: gaby@uis.edu.co
} 
1) La inmediata reconexión del ramal fallado, lo cual implica un riesgo para personas y equipo si la falla persiste.

2) Enviar personal calificado para verificar el estado de las líneas antes de hacer la reconexión, lo que implica una interrupción del servicio.

Este aspecto resalta la importancia del proceso de localización de este tipo de fallas [1].

Existen también fallas transitorias (por ejemplo: contacto con vegetación, arcos debido al desgaste del aislamiento de las líneas o daños en los aisladores), éstas, a diferencia de las permanentes, no se pueden localizar con una simple inspección. Ante estas fallas, un localizador permite detectar puntos débiles del sistema de potencia y tomar acciones correctivas para evitar problemas debidos a la reincidencia de la falla.

Varios métodos de detección incluyendo indicadores luminosos y relés a tierra están disponibles comercialmente. Sin embargo, la falta de una corriente significativa de falla y un cambio uniforme en la tensión de toda la red complican la búsqueda de la misma. Existen pocas técnicas de localización de este tipo de fallas [1]; la mayoría de los trabajos publicados acerca de la localización de fallas consideran las de baja impedancia [7, 12-15]. Los algoritmos para localización de fallas basados en la reactancia aparente [16], utilizados comúnmente en relés digitales, pueden introducir errores significativos en la localización de fallas de alta impedancia como se muestra en [13]. Los modernos relés numéricos tienen mejoras en protección de redes por estimación de fasores de tensión y corriente y el procesamiento digital de señales. Los algoritmos de procesamiento digital de señales incluidos en algunos relés mejoran el desempeño para la detección, identificación y localización de fallas. Otros métodos utilizan señales de alta frecuencia y ondas viajeras, que requieren una búsqueda exhaustiva utilizando medidores portátiles para rastrear la señal de registro inyectada desde la fuente con el fin de localizar la falla [17]. En general, todos estos métodos requieren la inserción de aparatos especializados representando un alto costo en la protección del sistema, además de la complejidad en la aplicación del método [17-19].

Ante estos problemas, en esta publicación se propone un método sencillo y eficaz de localización de fallas, aun de alta impedancia o en sistemas con sobrecarga. Este nuevo esquema parte de curvas de distancia bases de la impedancia aparente en el plano R-X del sistema ante diversas condiciones de falla, ubicando la falla mediante un valor de distancia. Las curvas de distancia son obtenidas de simulaciones ante diferentes escenarios de falla donde se emplean los parámetros del circuito y la distribución de carga, información requerida por los métodos comunes de localización de fallas basados en impedancia [19-24]. La falla se localiza a partir del valor de impedancia aparente calculado de los fasores de la componente fundamental de tensión y corriente estimados en la cabecera del circuito, obteniendo la distancia de ocurrencia en el sistema mediante interpolación en el plano R-X. Esta propuesta no requiere la utilización de instrumentos adicionales, sólo se necesita la medición de las señales de tensión y corriente en la cabecera del circuito para la ubicación de la falla.

En la segunda parte del artículo se presentan los fundamentos básicos del comportamiento de una falla en el diagrama $\mathrm{R}-\mathrm{X}$ y la metodología a seguir para localizarla; en el tercer apartado se muestran los resultados obtenidos con la metodología propuesta en un sistema prototipo y finalmente se presentan las conclusiones derivadas de esta investigación.

\section{IMPEDANCIA APARENTE DE UN CIRCUITO DE DISTRIBUCIÓN EN ESTADO DE FALLA}

Se define la impedancia aparente, como la impedancia medida desde la cabecera del circuito a partir de los fasores de la componente fundamental de tensión y corriente en el estado estable de la falla; es decir, que para un sistema trifásico se tienen seis impedancias:

$$
\begin{gathered}
Z_{\text {Aaparente }}=\frac{V_{A f}}{I_{A f}} \\
Z_{\text {Baparente }}=\frac{V_{B f}}{I_{B f}} \\
Z_{C_{\text {aparente }}}=\frac{V_{C f}}{I_{C f}} \\
Z_{A B_{\text {aparente }}}=\frac{V_{A f}-V_{B f}}{I_{A f}-I_{B f}} \\
Z_{B C_{\text {aparente }}}=\frac{V_{B f}-V_{C f}}{I_{B f}-I_{C f}} \\
Z_{C A_{\text {aparente }}}=\frac{V_{C f}-V_{A f}}{I_{C f}-I_{A f}}
\end{gathered}
$$

Los subíndices A, B y C indican las fases y la letra $\mathrm{f}$ el estado estable de la falla. Con las ecuaciones (1)-(6) 
se obtienen las impedancias aparentes vistas desde la cabecera del circuito.

El análisis de la impedancia aparente se realiza con las fases involucradas en la falla. Es decir, en caso de una falla monofásica, se analiza la impedancia aparente de la fase fallada, ecuaciones (1)-(3), mientras que en el caso de una falla bifásica o bifásica a tierra, se analiza el comportamiento de la impedancia aparente de línea de las fases involucradas en la falla, ecuaciones (4)-(6); y en el caso de una falla trifásica se analiza cualquier impedancia aparente de línea.

\section{Modelo simplificado de un ramal del sistema de distribución}

El modelo simplificado de un ramal de un sistema de distribución radial se muestra en la figura 1 , donde $\mathrm{Z}_{\mathrm{L}}$ es la impedancia serie de la línea, $Z_{C}$ es la impedancia de carga y $Z_{\text {pre }}$ es la impedancia vista en la subestación (en este caso $Z_{\text {pre }}=Z_{L}+Z_{C}$ ).

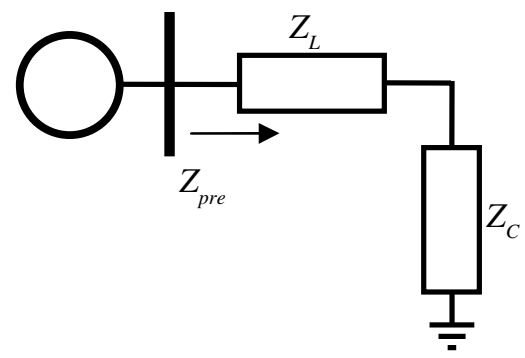

Figura 1. Modelo simplificado de un circuito de un sistema de distribución radial antes de la falla.

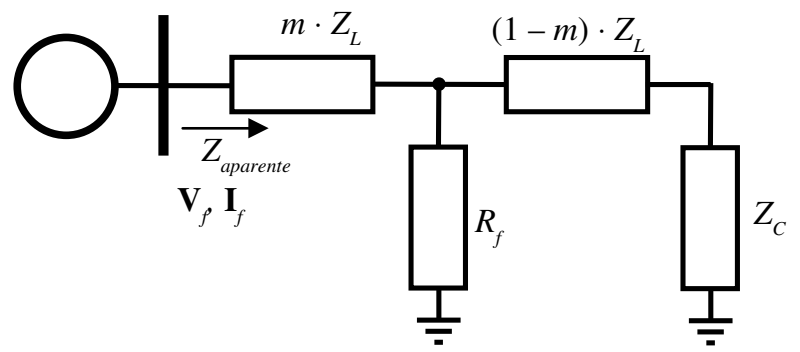

Figura 2. Modelo simplificado del circuito equivalente en un punto $m$ cuando ocurre una falla.

El circuito equivalente cuando ocurre una falla se muestra en la figura 2, donde la impedancia aparente (la impedancia vista desde la subestación), a partir de las medidas en el estado de falla es:

$$
Z_{\text {aparente }}\left(m, R_{f}\right)=\frac{V_{f}\left(m, R_{f}\right)}{I_{f}\left(m, R_{f}\right)} x
$$

Siendo $V_{l}\left(m, R_{1}\right)$ la tensión en estado estable de la falla medida en la cabecera del circuito e $I_{1}\left(m, R_{l}\right)$ la corriente en estado estable de la falla medida en la cabecera del circuito.

Esta impedancia también se puede expresar en términos de las impedancias mostradas en el circuito de la figura 2 como:

$$
Z_{\text {aparente }}\left(m, R_{f}\right)=m \cdot Z_{L}+\frac{R_{f} \cdot\left((1-m) \cdot Z_{L}+Z_{C}\right)}{R_{f}+(1-m) \cdot Z_{L}+Z_{C}}
$$

Donde $m$ es la fracción de la línea fallada $\mathrm{y}_{\mathrm{f}}$ es la resistencia de falla. Como se observa en la ecuación (8), el valor de la impedancia aparente es función de la distancia al punto de falla $m$ y la resistencia de la misma $\mathrm{R}_{\mathrm{f}}$, valores desconocidos cuando ocurre una falla.

\section{Curvas de distancia}

De acuerdo con la ecuación (8), ante una determinada configuración de carga, la impedancia aparente es función de la resistencia de falla y de la distancia donde ocurre ésta. A partir de esta ecuación se pueden obtener curvas de la impedancia aparente para determinados valores de $m$ (distancias), variando la resistencia de falla $\mathbf{R}_{\mathrm{f}}$, llamadas curvas de distancia. En la figura 3 se muestra de abajo hacia arriba el comportamiento de la impedancia aparente al 0, 30, 60 y 100\% de la longitud de la línea ${ }^{3}$, considerando 61 valores de la resistencia de falla entre 0 y $1000[\Omega]$.

En estas curvas de distancia se observa el efecto de la resistencia de falla en la impedancia aparente a una determinada distancia de la misma.

\footnotetext{
3 Se utiliza el circuito simplificado (figura 1), donde $Z_{L}$ y $Z_{C}$ son la impedancia del ramal principal y la impedancia de carga total del circuito mostrado en la figura 6 respectivamente.
} 


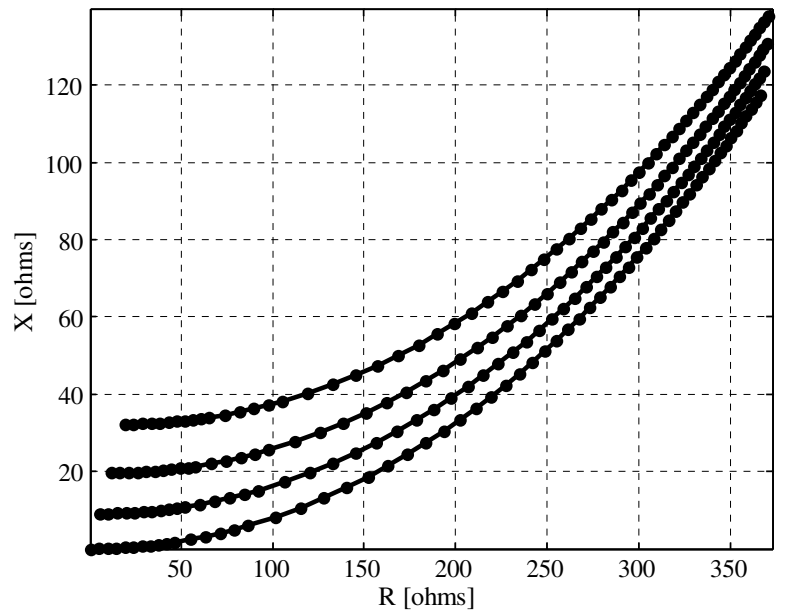

Figura 3. Curvas de distancia variando $R_{\mathrm{f}}$ de 0 a $1000[\Omega]$ a $0,30,60$ y $100 \%$ de la línea de abajo hacia arriba

\section{Curvas de resistencia de falla}

De igual forma a como se obtienen las curvas de distancia, se pueden obtener curvas de resistencia, para lo cual en la ecuación (8) se varía $m$ para determinados valores de $\mathrm{R}_{\mathrm{f}}$. En la figura 4 se muestra el comportamiento de la impedancia aparente para 21 resistencias de falla entre 0 y $1000[\Omega]$ variando la proporción de la línea fallada del 0 al $100 \%$. En estas curvas de resistencia se observa el efecto de la distancia a la falla en la impedancia aparente para una determinada resistencia de la misma.

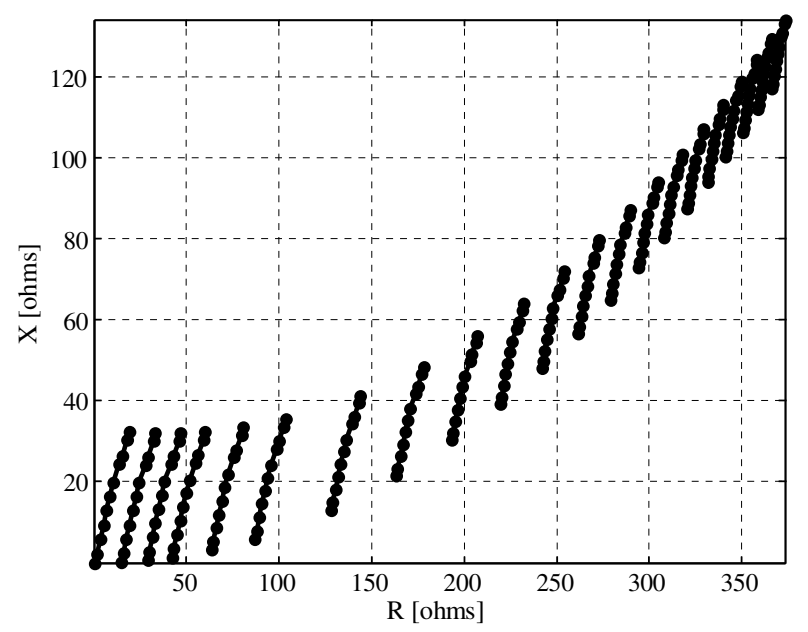

Figura 4. Curvas de resistencia variando $m$ del 0 al $100 \%$ de la línea a $0 ; 15 ; \ldots ; 950$ y $1000[\Omega]$ de izquierda a derecha.

\section{Circuito completo}

Por medio de simulaciones de un circuito en falla, se puede obtener la impedancia aparente de una distribución de carga para distintos valores de $\mathrm{R}_{\mathrm{f}} \mathrm{y} m$, teniendo en cuenta distintos modelos de carga y línea, desbalance del sistema, carga distribuida, entre otros.

En la figura 5 se comparan los resultados al utilizar el circuito simplificado (figura 1) y el circuito completo (figura 6). En las líneas continuas de la figura 5 se muestran las curvas de distancia obtenidas a partir del circuito completo y en las líneas punteadas se muestran las del circuito simplificado. Se puede observar cómo el error en la estimación de las impedancias (utilizando el circuito simplificado) aumenta para valores elevados de $m$ y $\mathrm{R}_{\mathrm{f}}$.

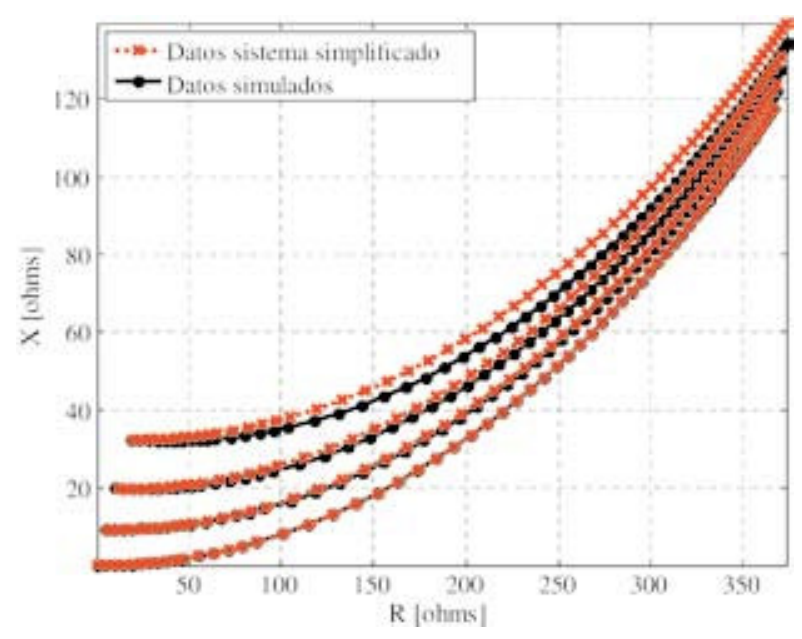

Figura 5. Curvas de distancia de los circuitos completo y simplificado variando $R_{\mathrm{f}}$ de 0 a $1000[\Omega]$ a 0, 30, 60 y $100 \%$ de la línea de abajo hacia arriba.

\section{Interpolación}

La interpolación consiste en hallar un dato dentro de un intervalo en el cual se conocen los valores de frontera (extremos). La interpolación lineal bidimensional, también conocida como interpolación lineal triangular, consiste en construir un plano que aproxime los valores contenidos en los límites de tres puntos conocidos para una región especificada en la intersección de las líneas que los unen, así ante las coordenadas de un nuevo punto de dos dimensiones se obtiene el valor del plano generado. En esta situación en particular se utiliza una interpolación lineal entre cada pareja de puntos, obteniendo una función definida por regiones y cada región definida por una función lineal. A continuación se establece cómo utilizar la interpolación para la localización de fallas en sistemas de distribución. 
1) Curvas de distancia: Ante el evento de una falla en una determinada fracción de una línea, se genera una curva de distancia con un comportamiento que responde a la variación en la resistencia de la falla, lo cual se refleja en la impedancia aparente del sistema donde ocurre la falla. Por lo tanto, para un valor de impedancia aparente correspondiente a una configuración de carga conocida, se puede estimar una distancia, puesto que cada curva de distancia tiene un valor específico de la fracción de línea $m$; sin embargo, la consideración de todas las posibles curvas de distancia es inadmisible, puesto que los posibles valores de $m$ son infinitos. Ante este hecho se propone la utilización de interpolación bidimensional con un número reducido de curvas de distancia que se obtienen por simulación del sistema. Dado un valor de impedancia aparente del sistema con falla, se estima la distancia de la falla medida desde la subestación, valiéndose del plano R-X y la interpolación bidimensional de curvas de distancia base obtenidas previamente de simulación para identificar la correspondiente curva de distancia y por ende la fracción de la línea donde ocurre la falla.

2) Curvas de resistencia: $\mathrm{Al}$ igual que la interpolación de las curvas de distancia, cuando ocurre una falla se puede obtener la resistencia a la falla a partir de las curvas de resistencia. La resistencia de falla aporta información sobre la naturaleza de la causa de la falla. La aplicación del método es análogo al tratado en este artículo de distancia.

\section{PRUEBAS Y RESULTADOS}

El circuito seleccionado para realizar las pruebas es el circuito radial de distribución SaskPower de 25[KV] que se encuentra en Saskatchewan (Canadá), mostrado en [20]. El diagrama unifilar del circuito se muestra en la figura 6. Este sistema ha sido utilizado para pruebas de distintos métodos de localización de fallas de baja impedancia [20-24]. A partir de datos obtenidos mediante simulaciones del sistema ante diferentes distancias de falla $(m)$ y resistencias de falla $\left(\boldsymbol{R}_{\mathrm{f}}\right)$, se construye una base de datos de las impedancias aparentes de las fallas realizadas en todas las barras del ramal principal (barras $1 \mathrm{a}$ 12) con 61 resistencias de falla entre 0 y $1000[\Omega]$, para a partir de éstas obtener las curvas de distancia. Debido a que se quiere mostrar el rendimiento del método propuesto, se utilizarán unas fallas para generar las curvas de distancia (curvas base) y los demás datos serán localizados por el método. Las curvas de distancia base que se utilizan para realizar la interpolación son de fallas en las barras
1,6 y 12 que coinciden con el 0,50 y $100 \%$ de la línea respectivamente. La demás barras $(2,3,4,5,8,9,10$ y 11) se utilizan para la evaluación del método.

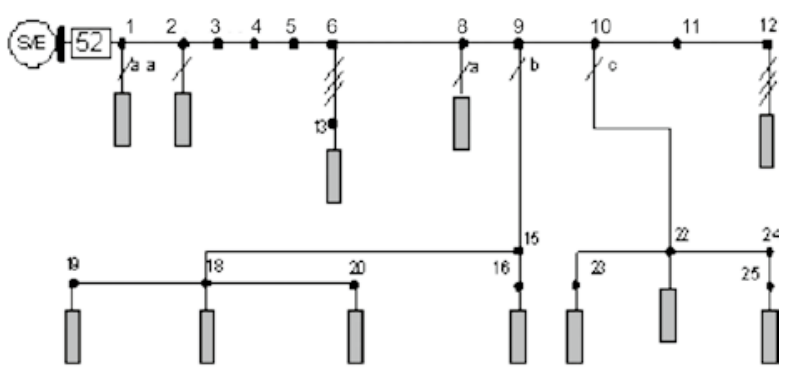

Figura 6. Circuito prototipo de SaskPower de 25[KV] que se encuentra en Saskatchewan (Canadá), con longitud de $37,013[\mathrm{Km}]$ en el ramal principal (del nodo 1 al 12).

En las figuras 7, 8, 9 y 10 se muestran las curvas de distancia de las impedancias aparentes ante una falla monofásica, bifásica, bifásica a tierra y trifásica respectivamente. En estas figuras se observa que los comportamientos de las impedancias aparentes son similares ante cualquier tipo de falla. Como se mencionó antes, se utilizan datos para construir las curvas base necesarias para realizar la interpolación (en las figuras líneas sólidas) y el resto de datos (líneas a trazos) son utilizados para evaluar el desempeño del método.

Se comparan las distancias obtenidas de la interpolación con las distancias reales en proporción a la longitud del ramal por medio de la ecuación de error:

$$
\% \text { error }=\frac{\text { distancia }_{\text {est }}-\text { distancia }_{\text {real }}}{\text { distancia }_{\text {total }}} \times 100
$$

Donde distancia $a_{\text {est }}$ es la distancia estimada por el método, distancia $_{\text {real }}$ es la distancia real al punto de falla y distancia $_{\text {total }}$ es la longitud total del ramal donde se está ubicando la falla. 


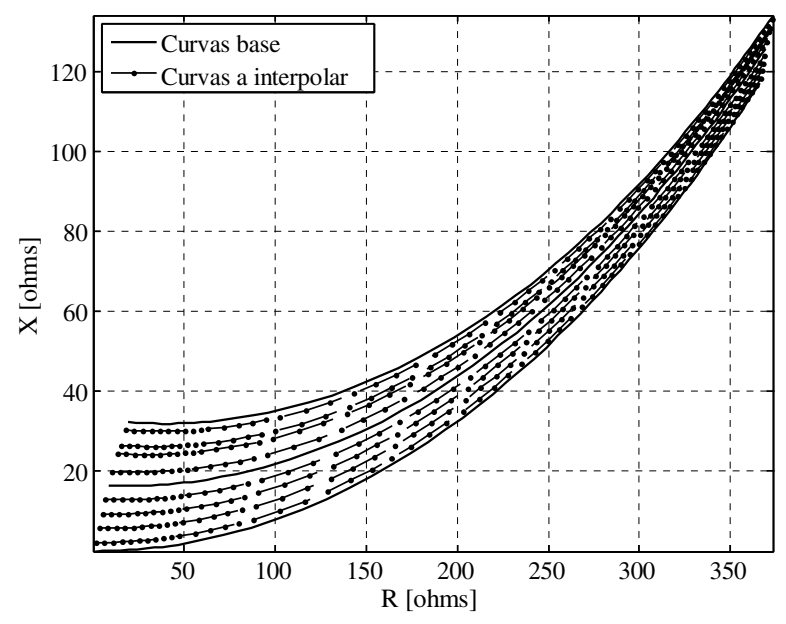

Figura 7. Curvas de distancia de una falla monofásica (a-t) en el ramal principal.

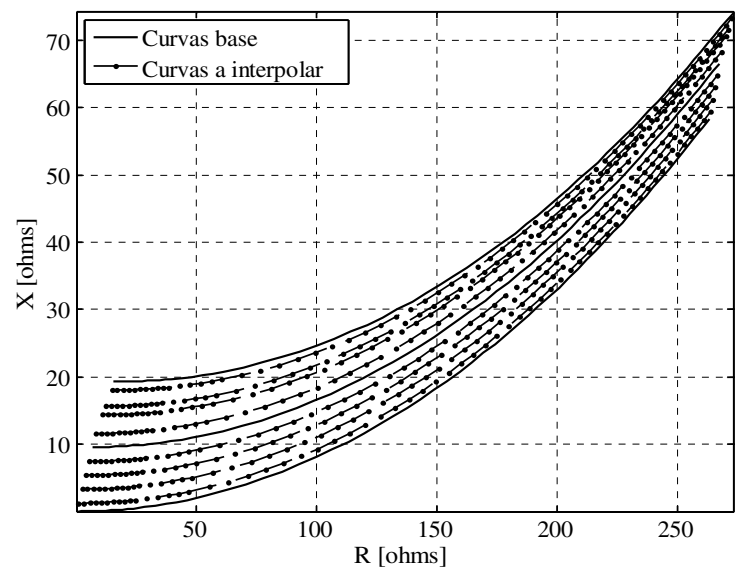

Figura 8. Curvas de distancia de una falla bifásica (b-c) en el ramal principal

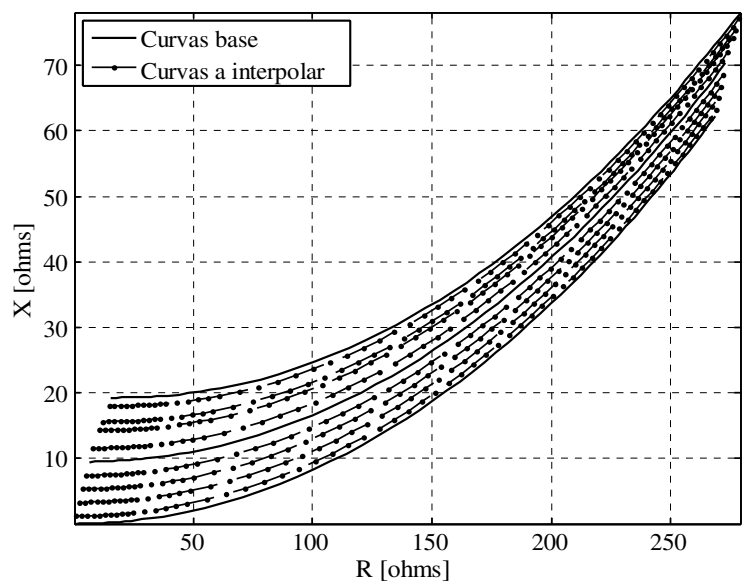

Figura 9. Curvas de distancia de una falla bifásica a tierra (c-a-t) en el ramal principal.

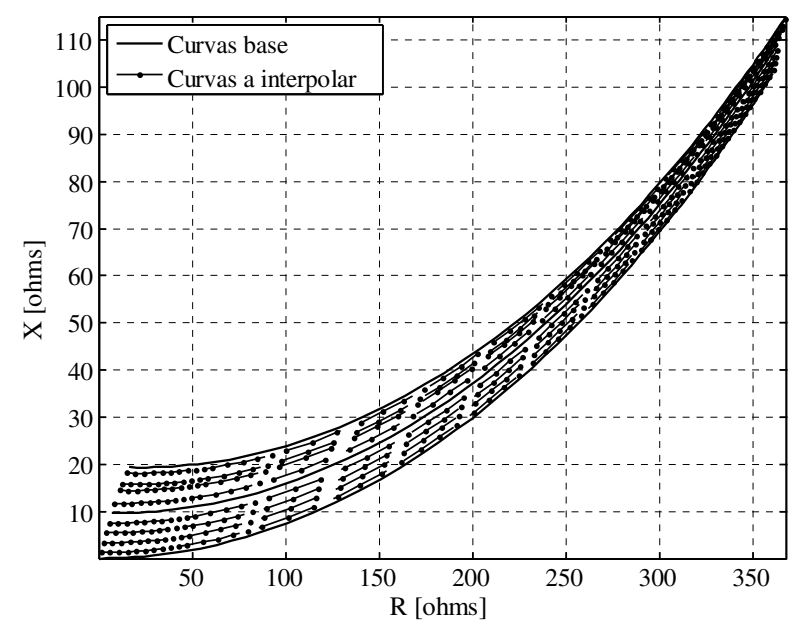

Figura 10. Curvas de distancia de una falla trifásica (a-b-c) en el ramal principal.

En las figuras 11, 12, 13, 14 y 15 se muestran los errores de localización del método propuesto según (9) ante resistencias de falla de $0,240,500,750$ y $1000[\Omega]$ respectivamente. Las fallas de $0[\Omega]$ son las que menor error presentan al localizarse (de aquí a que la mayoría de métodos se enfoquen en estos valores de resistencia), y las resistencia de falla de $1000[\Omega]$ es la que mayor error presenta. Para el resto de resistencias de falla, se tienen errores dentro de estos límites.

El error aumenta notablemente en las barras 8, 9 y 10 como se observa en las figuras 11 a 15 ; este hecho se debe principalmente a que estas barras tienen mayor carga, lo cual tiene una gran influencia en las curvas de resistencia reflejándose en una mayor curvatura (a diferencia del tramo entre las barra 1 a 5). Debido a que el método empleado está basado en interpolación lineal y la aproximación es realizada entre las barra 6 y 12, la curva de interpolación obtenida presenta mayor error en comparación con la curva de interpolación entre las barras 1 y 6 , ver figura 4 . Sin embargo, este inconveniente puede ser tratado al incluir más barras en la generación de las curvas base, pues en este caso se utilizaron pocas barras con la intención de probar el método con suficiente información. 


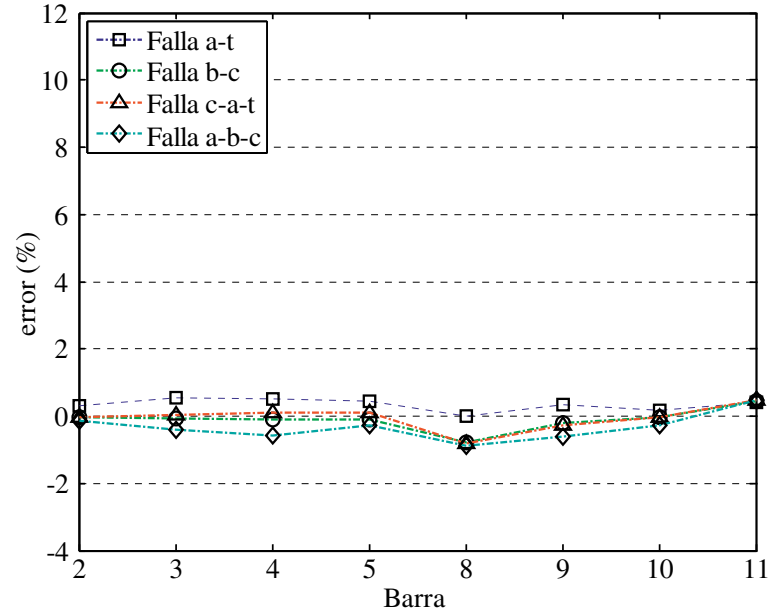

Figura 11. Errores de la localización de distintos tipos de falla con una resistencia de falla de $0[\Omega]$.

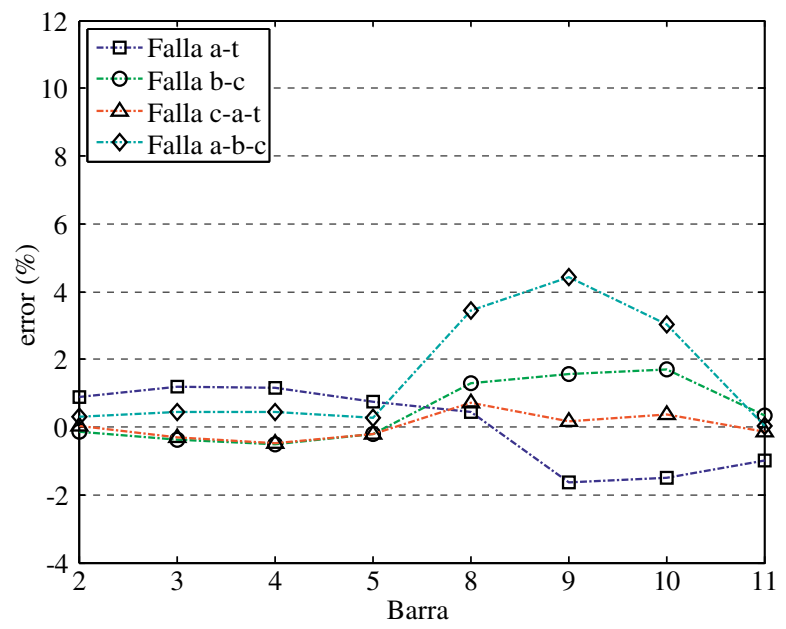

Figura 12. Errores de la localización de distintos tipos de falla con una resistencia de falla de $240[\Omega]$.

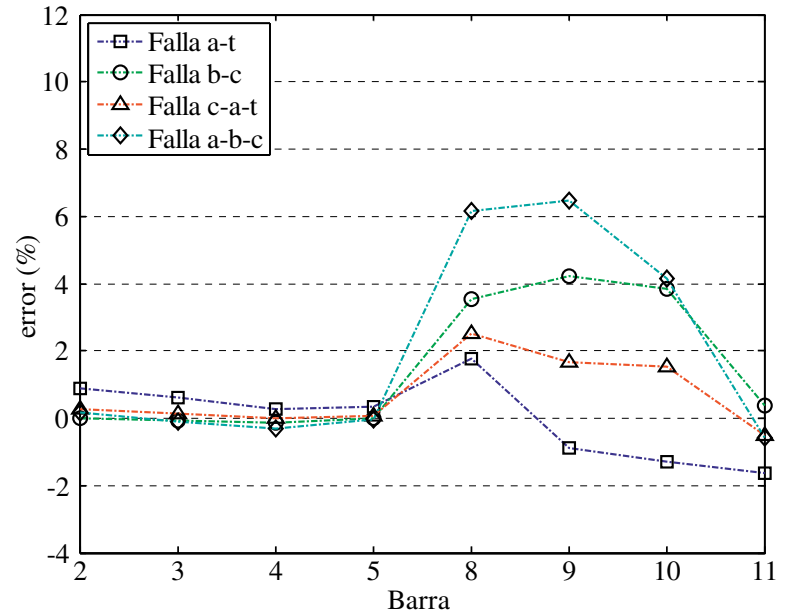

Figura 13. Errores de la localización de distintos tipos de falla con una resistencia de falla de $500[\Omega]$.

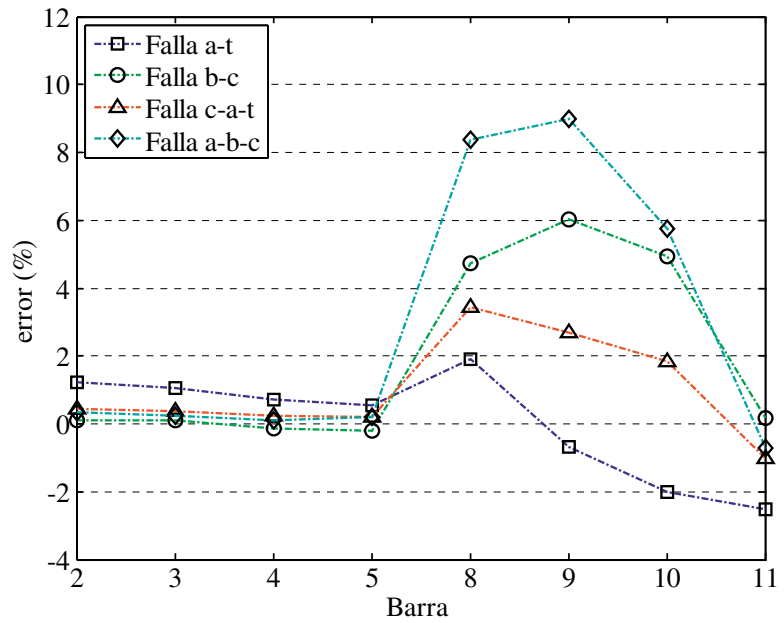

Figura 14. Errores de la localización de distintos tipos de falla con una resistencia de falla de $760[\Omega]$.

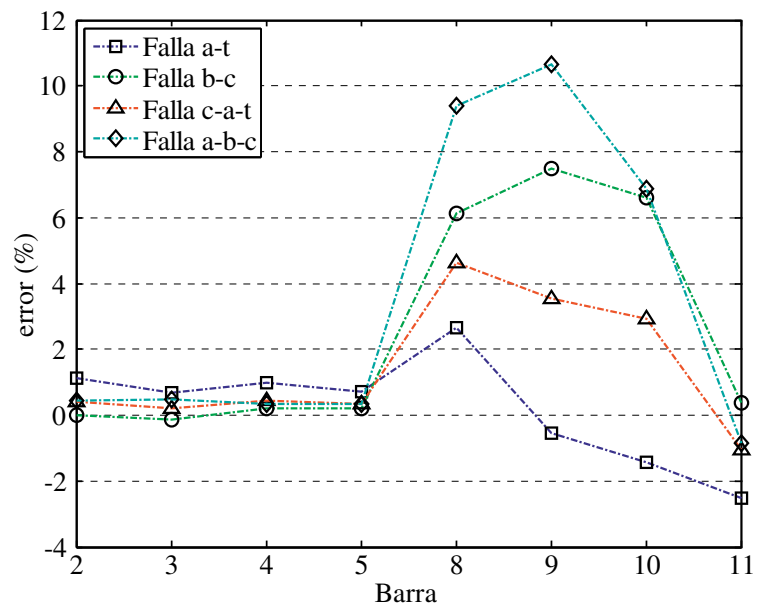

Figura 15. Errores de la localización de distintos tipos de falla con una resistencia de falla de $1000[\Omega]$. 
La metodología es aplicable de igual forma a los demás ramales, donde los errores son similares. Para localizar una falla, ésta se debe localizar por cada ramal, debido a que no se sabe en cuál ha ocurrido, entonces se tienen múltiples estimaciones de distancias de falla (una por cada ramal del sistema), este es un problema común en todos los métodos de distancia [25]. Existen múltiples metodologías para atacar el problema de la múltiple estimación con herramientas de clasificación de inteligencia artificial [26].

En [20] se hacen pruebas con el mismo circuito y se presentan errores alrededor del $3 \%$ ante resistencias de falla de máximo $50[\Omega]$ en el caso de falla monofásica. Cabe resaltar que al ir aumentando la resistencia de falla, mayor es el error. El método aquí propuesto, para el mismo tipo de falla, presenta errores siempre inferiores del 3\% (en el rango de resistencias de falla de análisis de 0 a $1000[\Omega])$ a excepción de resistencias de falla alrededor de los $1000[\Omega]$ (caso más crítico), donde el error supera ligeramente este valor. Se concluye que el método aquí propuesto presenta un desempeño excelente en fallas de baja impedancia (alrededor de los $50[\Omega]$ ), superando el desempeño de métodos como el presentado en [20], además de presentar resultados satisfactorios para el caso de fallas de alta impedancia.

\section{CONCLUSIONES}

Se presentó un método de localización de fallas de alta impedancia empleando impedancias aparentes para construir curvas de distancia y localizar fallas por medio de interpolación bidimensional. A diferencia de los métodos comúnmente utilizados en la localización de fallas de alta impedancia, se utilizan únicamente medidas fasoriales de la componente fundamental de tensión y corriente en la cabecera del circuito. Se simulan fallas en todas las barras del circuito con resistencias de falla de hasta $1000[\Omega]$, siendo aplicable esta metodología a valores mayores.

Para someter a prueba la metodología propuesta, se utilizaron alrededor de 500 datos de falla a distintas distancias y con resistencias de falla de hasta $1000[\Omega]$, donde en el peor de los casos se obtuvieron resultados con errores inferiores al 3, 5, 8 y $11 \%$ para el caso de falla monofásica, bifásica, bifásica a tierra y trifásica respectivamente, mostrando de esta manera el alto desempeño del método propuesto.

Se propone una metodología de fácil implementación y económica en comparación de los métodos comúnmente utilizados para localizar las fallas de alta impedancia; con la ayuda del método aquí propuesto se puede reducir considerablemente el tiempo de ubicación de la falla en un sistema, disminuyendo por lo tanto el tiempo de la interrupción del servicio con la consiguiente mejora de los índices de calidad (DES y FES en el caso colombiano).

\section{REFERENCIAS}

[1] T. Baldwin, F. Renovich and L. Saunders. "Directional ground-fault indicator for highresistance grounded systems". IEEE Transactions on Industry Applications. Vol. 39 N $^{\circ}$ 2, pp. 325-332. March/April 2003.

[2] F.M. Uriarte. "Modeling, Detection and Localization of High-Impedance Faults In Low-Voltage Distribution Feeders". Blacksburg, Virginia: Master of Science in Electrical Engineering. Virginia Tech Polytechnic Institute and State University. December 2006.

[3] A. Bretas, M. Moreto, R. Salim and L. Pires. "A novel high impedance fault location for distribution systems considering distributed generation". Transmission \& Distribution Conference and Exposition, IEEE, Latin America. 2006.

[4] L. Garcia, P. Bastard, M. Petit, I. Gal, E. Lopez and H. Opazo. "Down-conductor fault detection and location via a voltage based method for radial distribution networks". Proceedings IEE Generation, Transmission \& Distribution. Vol. 152 $\mathrm{N}^{\circ}$ 2, pp. 180-184. March 2005.

[5] B.D. Russel and R. P. Chinchali. "A digital signal processing algorithm for detection arcing faults on power distribution feeders". IEEE Transactions on Power Delivery. Vol. $4 \mathrm{~N}^{\circ}$ 1, pp. 132-140. January 1989.

[6] D.I. Jeerings and T.R. Linders. "A practical protective relay for down-conductor faults". IEEE Transactions on Power Delivery. Vol. $6 \mathrm{~N}^{\circ}$ 2, pp. 565-574. April 1991.

[7] P. Jarventausta, P. Verho, M. Karenlampi and J. Partanen. "AI-based methods in practical fault location of medium voltage distribution feeders". Proceedings ISAP 196. International Conference on Intelligent Systems Applications to Power System. January/February 1996. 
[8] K. Lien, S. Chen, C. Liao, T. Guo, T. Lin and J. Shen. "Energy variance criterion and threshold tuning scheme for high impedance fault detection". IEEE Transactions on Power Delivery. Vol. 4 No 3, pp. 810-817. July 1999.

[9] C.L. Benner and B.D. Russel. "Practical highimpedance fault detection on distribution feeders". IEEE Transactions on Industry Applications. Vol. 33 No 3, pp. 635-640. May/June 1997.

[10] F.G. Jota and P. R. S. Jota. "High-impedance fault identification using a fuzzy reasoning system". IEE Proceedings Generation, Transmission and Distribution. Vol. $145 \mathrm{~N}^{\circ}$ 6, pp. 656-661. November 1998.

[11] C.G. Wester. "High impedance fault detection on distribution systems". The 42nd Annual Conference. Rural Electric Power Conference. N ${ }^{\circ} 26-28$, pp. c5 - 1-5. April 1998.

[12] W.H. E. Liu and W. Zhong. "A fuzzy set method for fault location identification in power distribution systems". Proceedings of the 35th IEEE Conference on Decision and Control. Vol. $2 \mathrm{~N}^{\circ} 11-13$, pp. 22082212. December 1996.

[13] G. Eberl, S. Hnninen, M. Lehtonen and P. Schegner. "Comparison of artificial neural networks and conventional algorithms in ground fault distance computation". Proceedings IEEE Power Engineering Society Winter Meeting. Vol. 3 No 23-27, pp. 19911996. January 2000.

[14] H. Nouri, C. Wang and T. Davies. "An accurate fault location technique for distribution lines with tapped loads using wavelet transform". Proceedings IEEE Porto Power Tech Conference. Vol. 3, pp. 4, 10-13. September 2001.

[15] S.J. Lee, M.S. Choi, S.H. Kang, B.G. Jin, D.S. Lee, B.S. Ahn, N.S. Yoon, H.Y. Kim and S.B. Wee. "An intelligent and efficient fault location and diagnosis scheme for radial distribution systems". IEEE Transactions on Power Delivery. Vol. 19 $\mathrm{N}^{\circ}$ 2, pp. 524-532. April 2004.

[16] A. Warrington and C. Van. "Protective relays: Their theory and practice". Chapman and Hall Ltd. Vol. 2. Ed. 3, Cap. 6. London. 1969.
[17] L.F.S.T. Baldwin, F. Renovich and D. Lubkeman. "Fault locating in ungrounded and high-resistance grounded systems". IEEE Transactions on Industry Application. Vol. $37 \mathrm{~N}^{\mathrm{o}}$ 4, pp. 1152-1159. July/ August 2001.

[18] J.C. Das and R.H. Osman. "Grounding of AC and DC low voltage and medium voltage drive systems". Proceedings Annual Pulp on paper Industry Technical Conference IEEE. 16-20, pp. 36-47. June 1997.

[19] D.H. Lubich. "High resistance grounding and fault finding on three phase three wire (delta) power systems". Proceedings IEEE 1997 Annual Textile, Fiber, and Film Industry Technical Conference, 6-8, pp. 10. 5. May 1997.

[20] R. Das. "Determining the locations of faults in distribution systems". Ph.D. dissertation, University of Saskatchewan, Saskatoon, Canada. 1998.

[21] G. Morales, J. Mora, and S. Pérez. "Análisis del desempeño de un localizador de fallas basado en SVM ante la variación de carga en el sistema de distribución". Revista Scientia et Técnica (ISSN 0122-1701). Año XII No 32, pp. 7-12. Diciembre 2006. URLs: http://www.utp.edu.co/php/revistas/ ScientiaEtTechnica/docsFTP/1112337-12.pdf

[22] G. Morales, G. Carrillo and J. Mora. "Selección de descriptores de tensión para localización de fallas en redes de distribución de energía". Revista Ingeniería (ISSN 0121-750X), Vol. $11 \mathrm{~N}^{\circ} 1$, pp. 43-50. 2006.

[23] G. Morales, A. Gómez and H. Vargas. "Estrategia de mejoramiento en la selección de descriptores para la localización de fallas en sistemas de distribución con SVM". III Congreso Internacional de la Región Andina IEEE. Quito, Ecuador. Noviembre 2006.

[24] G. Morales, H. Vargas and J. Mora. "Impedance based method to fault location in power distribution, considering tapped loads and heavy unbalanced systems". XII encuentro regional Iberoamericano del CIGRÉ. Foz de Iguazú, Brasil. Mayo 2007.

[25] G. Morales, J. Mora and H. Vargas. "Método de localización de fallas en sistemas de distribución basado en gráficas de reactancia”. Revista Scientia 
et Técnica (ISSN 0122-1701). Vol. 34, pp. 49-54. Mayo 2007. URLs: http://www.utp.edu.co/php/ revistas/ScientiaEtTechnica/docsFTP/930349-54. pdf

[26] G. Morales, J. Mora and G. Carrillo. "Evaluación comparativa de tres métodos de clasificación aplicados al problema de la localización de fallas de cortocircuito en sistemas de distribución de energía eléctrica". Revista Scientia et Técnica (ISSN 0122-1701). Vol. 35, pp. 19-24. Agosto 2007. URLs: http://www.utp.edu.co/php/revistas/ ScientiaEtTechnica/docsFTP/10173719-24.pdf 